\title{
KÄHLERIAN METRICS GIVEN BY CERTAIN SMOOTH POTENTIAL FUNCTIONS
}

\author{
By Yoshiyuki Watanabe
}

\section{$\S 1$. Introduction.}

As is well known, every Kählerian metric $d s^{2}=2 \Sigma g_{\alpha \bar{\beta}} d z_{\alpha} d \bar{z}_{\beta}$ is locally expressible in the form

$$
g_{\alpha \bar{\beta}}=\frac{\partial^{2} \phi}{\partial z_{\alpha} \partial \bar{z}_{\beta}},
$$

with respect to local complex coordinates $\left\{z_{\alpha}\right\}, \alpha=1, \cdots, n$, where $\phi(z, \bar{z})$ is a real valued function of $\left\{z_{\alpha}, \bar{z}_{\alpha}\right\}$.

We now consider a Kählerian metric $g_{\alpha \bar{\beta}}$ with $\phi$ such that $\phi=f(t), t=\Sigma z_{\alpha} \bar{z}_{\alpha}$, where $t \rightarrow f(t) \in C^{\infty}(R)$. S. S. Eum [1] studied such a Kählerian metric with nonzero constant holomorphic curvature defined in the complex number space $C^{n}$, and showed it is Fubinian, i. e.,

$$
f(t)=\frac{1}{k} \log (k t+b)+c,
$$

where $k(\neq 0), b(>0)$ and $c$ are constant. A Kählerian manifold with constant holomorphic curvature is harmonic (cf. S. Tachibana [7]). For this reason, the present author [12] studied Kählerian manifolds, which are harmonic. On the other hand, P.F. Klembeck [2] has shown that the complex space $C^{n}$ admits a complete Kählerian metric $h$ with components

$$
h_{\alpha \bar{\beta}}=\frac{\partial^{2} f(t)}{\partial z_{\alpha} \partial \bar{z}_{\beta}}, \quad f(t)=\int_{0}^{\infty} \frac{1}{r} \log (1+r) d r,
$$

which has strictly positive curvature. The Kählerian manifold $\left(C^{n}, h\right)$ is harmonic at the origin 0 of $C^{n}$ (cf. $\S 3$ ). But the scalar curvature is not constant as we can directly compute. Therefore $\left(C^{n}, h\right)$ is not harmonic, because a harmonic Riemannian manifold is Einsteinian (cf. [4]). Thus we are very interested in a Kählerian metric locally expressed in the form

$$
g_{\alpha \tilde{\beta}}=\frac{\partial^{2} f(t)}{\partial z_{\alpha} \partial \bar{z}_{\beta}}, \quad t \rightarrow f(t) \in C^{\infty}(R),
$$

Received May 25, 1981 
with respect to local complex coordinates $\left\{z_{\alpha}\right\}$ (we can see another interesting example in [8]).

In the present paper, we shall study Kählerian metrics, which are locally expressible in the form (1.3) and satisfy one of the following conditions:

$$
R=\text { constant, }
$$

where $R$ is the scalar curvature.

$$
R_{\imath \jmath ; k ; l}-R_{\imath \jmath ; l ; k}=0,
$$

where $R_{\imath}$ is the Ricci tensor and (;) denotes the covariant differentiation.

The main theorem is

THEOREM 1. A Kählerian manifold $\left(D^{n}, g\right)$ given by (1.3) is flat or Fubinian if it satisfies the condition (A) where $D^{n}$ is $C^{n}$ or its star-shaped subdomain at the origin of $C^{n}$

Preliminary facts will be given in $\S 2$ following to Yano-Bochner's notation (cf. [14]). In $\S 3$, we show that the Kählerian metric given by (1.2) is harmonic at the origin 0 of $C^{n}$. In $\S 4$, we shall prove that a Kählerian manifold $\left(C^{n}, g\right)$ given by (1.3) is locally flat or Fubinian if it satisfy the condition (B). The last section is devoted to prove Theorem 1.

The author wishes to express his sincere thanks to Prof. S. Ishihara and Prof. S. Tachibana, who give him many valuable suggestions and guidances. $\mathrm{He}$ also would like to acknowledge the continuing guidances and encouragements of Prof. K. Takamatsu.

\section{$\S 2$. Pleliminaries.}

We agree to adopt the summation convention and the following ranges of indices throughout the paper:

$$
\begin{aligned}
& 1 \leqq \imath, \jmath, k, \cdots \leqq 2 n, \\
& 1 \leqq \alpha, \beta, \gamma, \cdots \leqq n .
\end{aligned}
$$

Consider an $n$ complex dimensional Kählerian manifold with metric

$$
d s^{2}=\Sigma g_{j k} d z_{j} d z_{k},
$$

where $\left\{z_{\alpha}\right\}$ are local complex coordinates and $\bar{z}_{\alpha}=z_{\bar{\alpha}}$ (=conjugate of $z_{\alpha}$ ). As the metric is Kählerian, $g_{j k}$ satisfy the following conditions:

$$
g_{\alpha \beta}=g_{\bar{\alpha} \bar{\beta}}=0,
$$

and (2.1) becomes 


$$
d s^{2}=2 \sum g_{\alpha \bar{\beta}} d z_{\alpha} d \bar{z}_{\beta}
$$

$g^{j k}$ satisfy the corresponding equations to (2.2). The Christoffel symbols $\Gamma_{j k}^{i}$ vanish except

$$
\Gamma_{\beta \gamma}^{\alpha}=g^{\alpha \bar{\varepsilon}} \frac{\partial g_{\beta \bar{\varepsilon}}}{\partial z_{\gamma}},
$$

and their conjugates. As to the curvature tensor $R_{j k l}^{\imath}$, only the components of the form $R_{\beta \gamma \bar{\delta}}^{\alpha}$ and $R^{\alpha}{ }_{\beta \bar{\gamma} \delta}$ and their conjugate can different from zero, and it holds that

$$
R_{\beta \gamma \tilde{\delta}}^{\alpha}=\frac{\partial \Gamma^{\alpha}{ }_{\beta \gamma}}{\partial \bar{z}_{\delta}}
$$

from which

$$
\begin{aligned}
& R_{\beta \bar{\gamma}}=R_{\beta \bar{\gamma} \alpha}^{\alpha}=-\frac{\partial \Gamma_{\beta \alpha}^{\alpha}}{\partial \bar{z}_{\gamma}}, \\
& R_{\beta \gamma}=R_{\bar{\beta} \bar{\gamma}}=0 .
\end{aligned}
$$

The scalar curvature $R=g^{j k} R_{j k}$ is $2 g^{\alpha \beta} R_{\alpha \bar{\beta}}$. A Kählerian manifold is called a space of constant holomorphic curvature if its curvature tensor satisfies

$$
R_{\alpha \bar{\gamma} \gamma \bar{\delta}}=\frac{R}{2 n(n+1)}\left(g_{\alpha \bar{\beta}} g_{\gamma \bar{\delta}}+g_{\alpha \bar{\delta}} g_{\gamma \bar{\beta}}\right) .
$$

Let $C^{n}$ be the complex number space with complex coordinates $\left\{z_{\alpha}\right\}$, and $D^{n}$ be $C^{n}$ or its star-shaped subdomain centered at the origin of $C^{n}$. Now we are going to compute the curvature tensor, the Ricci tensor and the scalar curvature from the Kählerian metric given by (1.3).

First from (1.3), we have

$$
g_{\alpha \bar{\beta}}=f^{\prime} \delta_{\alpha \beta}+f^{\prime \prime} \bar{z}_{\alpha} z_{\beta},
$$

where dashes mean differentiation with respect to $t$. As the metric is positive definite, the function we consider should satisfy

$$
f^{\prime}>0, f^{\prime}+t f^{\prime \prime}>0
$$

on $D^{n}$, because $g^{\alpha \bar{\beta}}$ are given by

$$
g^{\alpha \bar{\beta}}=\frac{1}{f^{\prime}}\left(\delta_{\alpha \beta}-\frac{f^{\prime \prime}}{f^{\prime}+t f^{\prime \prime}} z_{\alpha} \bar{z}_{\beta}\right) .
$$

From (2.4), we have

$$
\Gamma_{\beta \gamma}^{\alpha}=\frac{f^{\prime \prime}}{f^{\prime}}\left(\bar{z}_{\beta} \delta_{\alpha \gamma}+\bar{z}_{\gamma} \delta_{\alpha \beta}\right)+\sigma z_{\alpha} \bar{z}_{\beta} \bar{z}_{\gamma},
$$

where 


$$
\sigma(t)=\frac{f^{\prime} f^{\prime \prime \prime}-2 f^{\prime \prime 2}}{f^{\prime}\left(f^{\prime}+t f^{\prime \prime}\right)}
$$

(2.5) and some computations give the following equations (cf. [1], [8]):

and

$$
\begin{aligned}
R_{\beta \gamma \bar{\delta}}^{\alpha}= & \frac{f^{\prime} f^{\prime \prime \prime}-f^{\prime \prime 2}}{f^{\prime 2}} z_{\bar{\delta}}\left(\bar{z}_{\beta} \delta_{\alpha \gamma}+\bar{z}_{\gamma} \delta_{\alpha \beta}\right)+\frac{f^{\prime \prime}}{f^{\prime}}\left(\delta_{\beta \delta} \delta_{\alpha \gamma}+\delta_{\gamma \delta} \delta_{\alpha \beta}\right) \\
& +\sigma^{\prime} z_{\alpha} \bar{z}_{\beta} \bar{z}_{\gamma} z_{\delta}+\sigma z_{\alpha}\left(\bar{z}_{\beta} \delta_{\gamma \delta}+\bar{z}_{\gamma} \delta_{\beta \delta}\right)
\end{aligned}
$$

$$
R_{\beta \bar{\delta}}=\mu \delta_{\beta \delta}+\lambda \bar{z}_{\beta} z_{\delta},
$$

where $\lambda$ and $\mu$ are functions defined by

$$
\lambda=-\frac{(n+1)\left(f^{\prime} f^{\prime \prime \prime}-f^{\prime \prime 2}\right)}{f^{\prime 2}}-\sigma^{\prime} t-\sigma
$$

and

$$
\mu=-\frac{(n+1) f^{\prime \prime}}{f^{\prime}}-\sigma t
$$

As a direct consequence of (2.13) and (2.14) we get

$$
\mu^{\prime}=\lambda
$$

which is remarkable. The scalar curvature $R$ is given by

$$
R=\frac{2}{f^{\prime}}\left\{\lambda t+n \mu-\frac{t(\lambda t+\mu) f^{\prime \prime}}{f^{\prime}+t f^{\prime \prime}}\right\} \text {. }
$$

\section{§3. A Kählerian manifold being harmonic at only one point.}

Our purpose of this section is to show that the Kählerian manifold $\left(C^{n}, h\right)$ with metric $h$ given by (1.2) is harmonic at the origin $O$ of $C^{n}$. M. Itoh [3] has shown that in such a $\left(C^{n}, h\right)$ every geodesic parametrized by arc length emanating from $O$ is described by

$$
z_{\alpha}=A^{\alpha} \sinh (s), \quad \Sigma A^{\alpha} \bar{A}^{\alpha}=1\left(A^{\alpha}=\text { constant }\right)
$$

where $s$ is the geodesic distance measured from $O$ with respect to $h$. Then from (3.1), we have

$$
\Sigma z_{\alpha} \bar{z}_{\alpha}=\sinh ^{2} s .
$$

Differentiating (3.2) by $z_{\beta}$, we have

$$
\begin{aligned}
\bar{z}_{\alpha} & =2 \sinh (s) \cosh (s) \frac{\partial s}{\partial z_{\alpha}} \\
& =\sinh (2 s) \frac{\partial s}{\partial z_{\alpha}},
\end{aligned}
$$


from which

$$
h^{\alpha \bar{\beta}} \frac{\partial s}{\partial z_{\alpha}} \frac{\partial s}{\partial \bar{z}_{\beta}}=\frac{1}{4},
$$

because of

$$
h^{\alpha \bar{\beta}}=\frac{\sinh ^{2} s}{\log \left(\cosh ^{2} s\right)} \delta_{\alpha \beta}-\left\{\frac{1}{\log \left(\cosh ^{2} s\right)}-\operatorname{coth}^{2} s\right\} z_{\alpha} \bar{z}_{\beta} .
$$

Differentiating (3.3) by $\bar{z}_{\beta}$, we have

$$
\delta_{\alpha \beta}=2 \cosh (2 s) \frac{\partial s}{\partial \bar{z}_{\beta}} \frac{\partial s}{\partial z_{\alpha}}+\sinh (2 s) \frac{\partial^{2} s}{\partial z_{\alpha} \partial \bar{z}_{\beta}} .
$$

Multiplying this equation by $h^{\alpha \bar{\beta}}$ and taking account of (3.4), we obtain

$$
\frac{2(n-1) \sinh ^{2} s}{\log \left(\cosh ^{2} s\right)}=\cosh (2 s)+\sinh (2 s) \Delta s,
$$

since $\Delta_{s}=2 h^{\alpha \bar{\beta}} \frac{\partial^{2} s}{\partial z_{\alpha} \partial \bar{z}_{\beta}}$. Therefore it follows that

$$
\Delta s=\frac{(n-1) \tanh (s)}{2 \log (\cosh (s))}-\operatorname{coth}(2 s) .
$$

Let $(M, g)$ be an $n$-dimensional Riemannian manifold and $O$ a point of $M$. We denote by $s$ the geodesic distance measured from $O$ to the point in a neighborhood of $O$. If $\Delta s$ is a function of $s$ only, then $(M, g)$ is called to be harmonic at the point $O$. When $(M, g)$ is harmonic at any point, it is called a harmonic Riemannian manifold (cf. [4]).

As the right hand side of (3.5) is a function of $s$ only, $\left(C^{n}, h\right)$ is harmonic at the origin of $C^{n}$. However, $\left(C^{n}, h\right)$ is not harmonic, because it is not locally flat or locally Fubinian (See Theorem 1).

\section{§4. A kählerian metric satisfying the condition (B).}

Let $D^{n}$ be $C^{n}$ or its star-shaped subdomain centered at the origin $O$ of $C^{n}$. In this section, let $\left(D^{n}, g\right)$ be a Kählerian manifold with metric $g$ given by (1.3). Suppose that $\left(D^{n}, g\right)$ satisfies the condition $(\mathrm{B})^{1)}$ :

$$
R_{\imath \jmath ; k ; l}-R_{\imath \jmath ; l ; k}=0 \text {. }
$$

Then by the Ricci's formula, we have

$$
R_{h j} R_{\imath k l}^{h}+R_{i h} R_{j k l}^{h}=0,
$$

1) This condition was studied by K. Sekigawa [5], K. Sekıgawa and H. Takag1 [6], H. Takagi [9], S. Tanno and other authors.

2) The author is grateful to Prof. S. Tanaka with whom the author had several conversations on differential equations in this section. 
from which

$$
R_{\alpha j} R_{\imath k l}^{\alpha}+R_{\bar{\alpha} j} R^{\bar{\alpha}}{ }_{\imath k l}+R_{\imath \alpha} R^{\alpha}{ }_{j k l}+R_{\imath \bar{\alpha}} R^{\bar{\alpha}}{ }_{j k l}=0 .
$$

Thus (4.1) is equivalent to

$$
R_{\alpha \bar{\lambda}} R_{\beta \gamma \gamma \bar{\delta}}^{\alpha}+R_{\beta \bar{\alpha}} R^{\bar{\alpha}}{ }_{\bar{\lambda} \gamma \bar{\delta}}=0 \text { (conj.), }
$$

by virtue of (2.5) and (2.6).

Now substituting (2.11) and (2.12) into the left hand side of (4.2), we can see that it reduces to the following (cf. Y. Watanabe [13], p. 79).

$$
\left\{\frac{\lambda f^{\prime \prime}}{f^{\prime}}+t \lambda \sigma+\mu \sigma-\frac{\mu\left(f^{\prime} f^{\prime \prime \prime}-f^{\prime \prime 2}\right)}{f^{\prime 2}}\right\}\left(z_{\alpha} \bar{z}_{\gamma} \delta_{\beta \delta}-\bar{z}_{\beta} z_{\delta} \delta_{\alpha \gamma}\right)=0 .
$$

Now we assume that $n \geqq 2$. Then we have

$$
\frac{\lambda f^{\prime \prime}}{f^{\prime}}+t \lambda \sigma+\mu \sigma-\frac{\mu\left(f^{\prime} f^{\prime \prime \prime}-f^{\prime \prime 2}\right)}{f^{\prime 2}}=0,
$$

taking account of $f(t) \in C^{\infty}(R)$. (4.3) gives

$$
\lambda f^{\prime} f^{\prime \prime}+t \lambda \sigma f^{\prime 2}+\mu \sigma f^{\prime 2}-\mu\left(f^{\prime} f^{\prime \prime \prime}-2 f^{\prime \prime 2}\right)-\mu f^{\prime \prime 2}=0,
$$

which implies

$$
\lambda f^{\prime} f^{\prime \prime}+t \lambda \sigma f^{\prime 2}-t \mu \sigma f^{\prime} f^{\prime \prime}-\mu f^{\prime \prime 2}=0,
$$

because of (2.10). Thus we obtain

$$
\left(f^{\prime \prime}+t \sigma f^{\prime}\right)\left(\lambda f^{\prime}-\mu f^{\prime \prime}\right)=0 .
$$

In the followings, we consider two cases, i. e., Case I where $f^{\prime \prime}+t f^{\prime} \sigma=0$ and Case II where $\lambda f^{\prime}-\mu f^{\prime \prime}=0$.

Case I: We assume that $f^{\prime \prime}+t \sigma f^{\prime}=0$ in an open subdomain $\Delta_{1}^{n}$ of $D^{n}$. Taking account of (2.10), we have

from which

$$
\frac{t f^{\prime}\left(f^{\prime} f^{\prime \prime \prime}-2 f^{\prime \prime 2}\right)}{f^{\prime \prime}\left(f^{\prime}+t f^{\prime \prime}\right)}+f^{\prime \prime}=0
$$

$$
t f^{\prime} f^{\prime \prime \prime}+f^{\prime} f^{\prime \prime}-t f^{\prime \prime 2}=0 .
$$

Putting $u=f^{\prime}$ in (4.5), we have

$$
t u u^{\prime \prime}+u u^{\prime}-t u^{\prime 2}=0 .
$$

The general solution of (4.6) is given by

$$
u=b t^{a},
$$

where $a$ and $b$ are integral constants. Therefore the general solution of (4.5) is given by 


$$
f=\frac{b}{a+1} t^{a+1}+c
$$

where $a, b$ and $c$ are integral constants. From (2.8) it is easily seen that $a=0$ and $b>0$ where $\Delta_{1}^{n} \ni O$. In this case the corresponding Kählerian metric $g$ is flat, i. e.,

$$
f=b t+c \quad(b>0)
$$

when $\Delta_{1}^{n} \ni O$.

Case II: Suppose that

$$
\lambda f^{\prime}-\mu f^{\prime \prime}=0
$$

holds in a subdomain $\Delta_{2}^{n}$ of $D^{n}$. First by (2.7) and (2.12) we see that the potential function satisfying (4.9) gives an Einsteinian metric. By (2.13) and (2.14), we have

$$
f^{\prime \prime}\left\{(n+1) f^{\prime \prime}+t \sigma f^{\prime}\right\}=(n+1)\left(f^{\prime} f^{\prime \prime \prime}-f^{\prime \prime 2}\right)+t \sigma^{\prime} f^{\prime 2}+\sigma f^{\prime 2},
$$

from which

$$
(n+1)\left(2 f^{\prime \prime 2}-f^{\prime} f^{\prime \prime \prime}\right)=\left(t \sigma^{\prime}+\sigma\right) f^{\prime 2}-t \sigma f^{\prime} f^{\prime \prime} .
$$

Taking account of (2.10), we have

$$
t f^{\prime} \sigma^{\prime}+\left\{(n+2) f^{\prime}+n t f^{\prime \prime}\right\} \sigma=0 .
$$

If $\Delta_{2}^{n}$ contains the origin $O$, putting $t=0$ in (4.11) we have

$$
\sigma(0)=0,
$$

because of $f^{\prime}(0)>0$.

If $t>0$, then multiplying (4.11) by $t^{n+1}\left(f^{\prime}\right)^{n-1}$, we have

$$
t^{n+2}\left(f^{\prime}\right)^{n} \sigma^{\prime}+(n+2) t^{n+1}\left(f^{\prime}\right)^{n} \sigma+n t^{n+2}\left(f^{\prime}\right)^{n-1} f^{\prime \prime} \sigma=0,
$$

from which, integrating

$$
t^{n+2}\left(f^{\prime}\right)^{n} \sigma=c,
$$

where $c$ is an integral constant. Since the function $f$ satisfies (4.9), by the argument of continuity of the left hand side of (4.12), we can conclude that $c=0$ if $\Delta_{2}^{n}$ contains the origin $O$. Thus we have

$$
\sigma(t)=0,
$$

together with (4.12) if $\Delta_{2}^{n} \ni O$. Moreover since

$$
\left(\frac{f^{\prime \prime}}{f^{\prime 2}}\right)^{\prime}=\frac{f^{\prime} f^{\prime \prime \prime}-2 f^{\prime \prime 2}}{f^{\prime 3}},
$$


(4.14) has two solutions: One is $f_{1}=a t+b$ where $a(>0)$ and $b$ are constant and the other $f_{2}=\frac{1}{k} \log (k t+c)+d$ where $k(\neq 0)$ and $c(>0)$ and $d$ are constants, that is, when $O \in \Delta_{2}^{n}$,

$$
f_{1}=a t+b \quad(a>0)
$$

or

$$
f_{2}=\frac{1}{k} \log (k t+c)+d \quad(k \neq 0, c>0) .
$$

Note that in the case of (4.14)" the corresponding Kählerian manifold $\left(\Delta_{2}^{n}, g\right)$ is of constant holomorphic curvature $k$ (see S. S. Eum [1]).

Finally the function $f$ given by (4.8) does not satisfy (4.13) with $c \neq 0$ and can be smoothly connected only the solution $f=b t+c(b>0)$ of $(4.14)^{\prime}$. Conversely the solution $f=\frac{1}{k} \log (k t+c)+d(k \neq 0, c>0)$ of (4.14)" does not satisfy (4.7).

Thus we obtain the following

Theorem 2. Let $D^{n}$ be $C^{n}(n \geqq 2)$ or its star-shaped subdomain containing the origin $O$ of $C^{n}$. Suppose that the Kählernan metruc given by (1.3) satisfies the condition (4.1). Then it is flat or Fubrnian.

\section{Proof of Main Theorem.} have

By assumption $R$ is constant in (2.16). Multiplying (2.16) by $f^{\prime}\left(f^{\prime}+t f^{\prime \prime}\right)$, we

$$
\frac{R}{2} f^{\prime}\left(f^{\prime}+t f^{\prime \prime}\right)=t \lambda f^{\prime}+n f^{\prime} \mu+(n-1) t f^{\prime \prime} \mu .
$$

Putting $t=0$ in (5.1), we have

$$
\frac{R}{2} f^{\prime}(0)-n \mu(0)=0,
$$

because of $f^{\prime}(0)>0$. For $t>0$, we multiply (5.1) by $t^{n-1}\left(f^{\prime}\right)^{n-2}$. Then taking account of (2.15), we have

from which

$$
\begin{aligned}
& \frac{R}{2}\left\{\left(f^{\prime}\right)^{n} t^{n-1}+t^{n}\left(f^{\prime}\right)^{n-1} f^{\prime \prime}\right\} \\
& =t^{n}\left(f^{\prime}\right)^{n-1} \mu^{\prime}+n t^{n-1}\left(f^{\prime}\right)^{n} \mu+(n-1) t^{n}\left(f^{\prime}\right)^{n-1} f^{\prime \prime} \mu,
\end{aligned}
$$

$$
\frac{R}{2 n} t^{n}\left(f^{\prime}\right)^{n}=t^{n}\left(f^{\prime}\right)^{n-1} \mu+C,
$$

where $C$ is an integral constant. Then we have 


$$
t^{n}\left(f^{\prime}\right)^{n-1}\left\{\frac{R}{2 n} f^{\prime}-\mu\right\}=C \text {. }
$$

But we can see that $C=0$, taking limit of the left hand side of (5.3) of $t$ as $t$ tends to 0 . Therefore we have

$$
\frac{R}{2 n} f^{\prime 2}\left(f^{\prime}+t f^{\prime \prime}\right)+(n+1) f^{\prime} f^{\prime \prime}+t f^{\prime} f^{\prime \prime \prime}+(n-1) t f^{\prime \prime 2}=0,
$$

Putting $t=0$ in (5.5), we have

$$
f^{\prime \prime}(0)+\frac{R}{2 n(n+1)} f^{\prime}(0)^{2}=0 .
$$

Multiplying (5.5) by $\left(f^{\prime}\right)^{n-2} t^{n}$, we have

from which

$$
\begin{aligned}
& \frac{R}{2 n}\left\{\left(f^{\prime}\right)^{n+1} t^{n}+\left(f^{\prime}\right)^{n} t^{n+1} f^{\prime \prime}\right\}+(n+1)\left(f^{\prime}\right)^{n-1} t^{n} f^{\prime \prime} \\
& +t^{n+1}\left(f^{\prime}\right)^{n-1} f^{\prime \prime \prime}+(n-1) t^{n}\left(f^{\prime}\right)^{n-2} f^{\prime \prime 2}=0,
\end{aligned}
$$

$$
t^{n+1}\left(f^{\prime}\right)^{n-1}\left\{f^{\prime \prime}+\frac{R}{2 n(n+1)} f^{\prime 2}\right\}=\widetilde{C},
$$

where $\tilde{C}$ is an integral constant. But we have

$$
\widetilde{C}=0
$$

taking limit of the left hand side of (5.7) of $t$ as $t$ tends to 0 . Thus we have

$$
f^{\prime \prime}+\frac{R}{2 n(n+1)} f^{\prime 2}=0 \text {. }
$$

together with (5.6).

Now if $R=0$ in (5.8), then $f^{\prime \prime}=0$, i. e., $f^{\prime}=$ constant. Hence it follows that the corresponding Kählerian metric is flat. If $R \neq 0$ in (5.8), then the corresponding Kählerian metric is Fubinian and the holomorphic curvature is $\frac{R}{2 n(n+1)}$ (cf. (2.11)) because of an elementary calculation. This proves the theorem.

By Theorem 1, we have the following

Corollary 3. Let $(M, g)$ be a complete Kählerian manifold. Suppose that $g$ is expressed in the form (1.3) and its scalar curvature $R$ is non-positive constant. Then $(M, g)$ is the unitary space $C^{n}$ or the complex hyperbolic space $H^{n}$ according as $R=0$ or $R<0$.

\section{BIBLIOGRAPHY}

[1] S. S. Eum, Notes on Kählerian metric, Kyungpook Math. J., 1 (1958), 13-21.

[2] P.F. KLEMBeCK, A complete Kählerian metric of positive curvature on $C^{n}$, Proc. 
Amer. Math., 64 (1977), 313-316.

[3] M. Iтон, Kählerian manifolds with curvature bounded from above by a decreasing function, Proc. Amer. Math. Soc., 75 (1979), 289-293.

[4] H.S. Ruse, A. G. Walker and T. J. Willmore, Harmonic Spaces, Roma, 1961.

[5] K. Sekigawa, Some hypersurfaces in a Euclidean space, Sci. Rep. Niigata Univ., 8 (1971), 19-25.

[6] K. SeKigawa AND H. TAKAgI, Some conformally flat spaces satisfying a certain condition on the Ricci tensor, Tôhoku Math. J., 23 (1971), 151-162.

[7] S. Tachibana, On the characteristic function of spaces of consttant holomorphic curvature, Colloq. Math. 26 (1972), 149-155.

[8] S. TAChibana AND R. C. Liu, Notes on Kählerian metrics with vanishıng Bochner curvature tensor, Kōdai Math. Sem. Rep. 22 (1970), 313-321.

[9] H. TAKAGI, An example of Riemannian manifolds satisfying $R(X, Y) \cdot R=0$ but not $\nabla R \neq 0$, Tôhoku Math. J., 24 (1972), 105-108.

[10] H. TAKAgi AND Y. Watanabe, Kählerıan manifolds with vanishing Bochner curvature tensor satisfying $R(X, Y) \cdot R_{1}=0$, Hokkaido Math. J., 3 (1974), 129-132.

[11] S. TANNo, Hypersurfaces satisfying a certain condition on the Ricci tensor, Tôhoku Math. Journ., 21 (1969), 297-303.

[12] Y. WAtANABE, On the characteristic function of harmonic Kählerian spaces, Tôhoku Math. Journ., 27 (1975), 13-24.

[13] Y. Watanabe, Notes on Kählerian metrics on domains in $C^{n}$, Math. Rep. Toyama Univ., vol. 1 (1978), 75-83.

[14] K. Yano And S. Bochner, Curvature and Bettı number, Ann. of Math. Stud 32 (1953).

Department of MATHEMATics

Toyama University

TOYAMA, JAPAN 